Revue de droit comparé du travail et de la sécurité sociale

2| 2019

L'ubérisation du travail

\title{
La jurisprudence américaine et l'ubérisation du travail
}

Allison Fiorentino

\section{(2) OpenEdition}

1 Journals

Édition électronique

URL : https://journals.openedition.org/rdctss/1552

DOI : 10.4000/rdctss. 1552

ISSN : 2262-9815

Éditeur

Centre de droit comparé du travail et de la sécurité sociale

Édition imprimée

Date de publication : 1 juin 2019

Pagination : 32-39

ISSN : 2117-4350

Référence électronique

Allison Fiorentino, «La jurisprudence américaine et l'ubérisation du travail », Revue de droit comparé du travail et de la sécurité sociale [En ligne], 2 | 2019, mis en ligne le 01 novembre 2021, consulté le 11 novembre 2021. URL : http://journals.openedition.org/rdctss/1552 ; DOI : https://doi.org/10.4000/ rdctss. 1552

\section{(c) (i) (9)}

Revue de droit comparé du travail et de la sécurité sociale est mise à disposition selon les termes de la Licence Creative Commons Attribution - Pas d'Utilisation Commerciale - Pas de Modification 4.0 International. 


\section{LA JURISPRUDENCE AMÉRICAINE ET L'UBÉRISATION DU TRAVAIL}

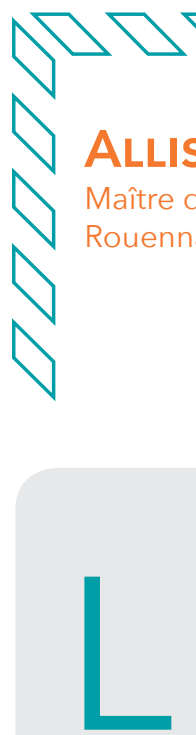

a distinction entre travailleur indépendant et salarié est un thème récurrent dans le contentieux prud'homal dans la mesure où le contrat de travail est la clé de voûte du droit social et commande son application. Le développement de la technologie qui a permis l'émergence de plateformes Internet et d'applications mobiles a donné un nouveau souffle à cette question. Des acteurs économiques tels qu'Uber, Deliveroo, Lyft, Amazon Mechanical Turk, Freelancer.com sont a priori seulement des intermédiaires mettant en contact deux contractants potentiels. En réalité, il s'agit d'acteurs économiques appelés à jouer un rôle de plus en plus important dans la vie des travailleurs. Selon un sondage du renommé Institut Gallup ${ }^{1}$, plus du tiers (36\%) des travailleurs américains sont concernés par cette nouvelle forme d'emploi, ce qui représente 57 millions de personnes.

Avec la montée en puissance de ces plateformes, de plus en plus de travailleurs y ont recours afin de bénéficier d'un complément de salaire. Les profils de ces travailleurs sont très divers : certains y ont recours entre deux CDI, d'autres durant des mises à pied sans rémunération, d'autres encore pour augmenter leur revenus en ayant recours à la flexibilité offerte par ces employeurs. Ces derniers y trouvent leur intérêt car la popularité croissante de l'économie collaborative, en tant que source ponctuelle de travail, permet à certaines sociétés de se constituer un vivier de travailleurs sans cesse renouvelé et sans rien leur devoir en cas de rupture de la relation contractuelle.

1 S. Mcfeely, R. Pendell, "What Workplace Leaders Can Learn From the Real Gig Economy ", 16 août 2018, https://www.gallup.com/workplace/240929/workplaceleaders-learn-real-gig-economy.aspx 
En effet, partant du principe que les travailleurs de ces sociétés sont des indépendants, celles-cin'offrent aucun des avantages auquel peut prétendre un salarié : l'assurance maladie, les régimes de retraite et les congés payés. Cela permet également aux entreprises d'éviter de payer les charges sociales, l'assurance chômage et l'assurance contre les accidents du travail. Cette qualification est donc particulièrement lucrative pour les employeurs, mais elle rencontre actuellement une résistance de plus en plus forte de leur main d'œuvre qui intente des actions en justice afin d'obtenir une requalification de leur relation de travail en salariat.

Les demandes en requalification sont intentées de deux manières. Soit une demande est déposée par un particulier devant une instance judiciaire étatique ou fédérale alléguant la violation de droits réservés aux salariés tels que le paiement des heures supplémentaires, soit un organisme public est saisi car, dans certains Etats, la qualification de salarié ou d'indépendant intéresse directement l'administration du travail. Les sommes réclamées à titre de dommages et intérêts sont particulièrement importantes en raison de la possibilité d'intenter une action de groupe.

La difficulté à laquelle se heurtent les demandeurs est la divergence de définitions concernant le statut de salarié. Chaque Etat a adopté la sienne, que ce soit du fait d'une jurisprudence ou d'une décision administrative. Bien qu'il existe un point commun entre ces définitions (le degré de contrôle de l'entreprise sur le travailleur), elles font appel à des critères différents de sorte qu'il est plus ou moins difficile pour les travailleurs d'une entreprise de faire valoir leur statut de salarié suivant l'Etat dans lequel ils travaillent.

En outre, certains textes fédéraux accordant des droits aux salariés comportent des définitions qui leur sont propres. II en va ainsi du National Labor Relations Act qui accorde des garanties concernant la négociation collective. Afin de pouvoir y prétendre, le travailleur doit satisfaire la définition suivante : il doit s'agir d'« une personne employée pour exécuter des prestations de service au profit d'autrui et qui, dans l'exercice de son activité, est soumise au contrôle de l'autre partie $»^{2}$. A cette définition légale sont ajoutés 11 critères facultatifs qui constituent autant d'indices de l'existence d'une relation salariale sans être pour autant déterminants. Parmi ces indices : l'étendue du contrôle exercé sur la prestation de travail, la durée de l'engagement, la question de savoir si le travail est effectué avec les outils de l'entreprise...

L'émergence de ce contentieux fera l'objet d'une première partie (I) avant d'analyser les changements jurisprudentiels (II) et, enfin, les possibles évolutions (III).

\section{I - L'ÉMERGENCE DU CONTENTIEUX DE L'ÉCONOMIE PARTICIPATIVE}

Ces dernières années, les demandes en justice se sont multipliées dans les Etats de New York et de Californie, de manière assez peu surprenante dans la mesure où le droit du travail est relativement favorable aux salariés. II était donc de l'intérêt des requérants de faire admettre par une juridiction de cet Etat l'existence d'une relation salariée. Afin de déterminer si un travailleur est correctement qualifié, les deux États se concentrent sur le niveau de contrôle que la société détient sur lui. La Californie utilisait jusqu'à peu, le critère

2 Cette définition ne figure pas dans la loi elle-même, mais l'organisme chargé du contrôle de la loi, le National Labor Relations Board, a recours à la définition de salarié contenue à l'article 220 du Restatement (Second) of Agency. 
de la réalité économique (également appelé test de contrôle multifactoriel) qui met l'accent sur le droit de l'employeur de contrôler le salarié, du point de vue du travail effectué et de la manière dont il est exécuté, et prend également en compte un certain nombre de facteurs. Les Etats de New York utilisent une approche similaire. Pour déterminer si le travailleur est indépendant, il faut établir qu'il est exempté de supervision, de direction et de contrôle dans l'exercice de ses fonctions en tenant compte d'un certain nombre de facteurs, tels que le fait qu'il détermine son propre horaire et offre ses services à d'autres entreprises.

Les premières affaires ne donnèrent pas lieu à des décisions dans la mesure où les requérants acceptèrent de transiger, mettant le juge dans l'impossibilité d'adapter sa jurisprudence à l'économie participative. Toutefois, l'importance des sommes versées par les sociétés démontre que cette question est appelée à devenir un enjeu majeur de la jurisprudence.

Ainsi, en septembre $2013^{3}$ par exemple, plusieurs conducteurs de Lyft intentèrent une action de groupe, alléguant qu'ils avaient été qualifiés à tort d'entrepreneurs indépendants et demandant un rappel de salaire et une indemnisation au titre des frais professionnels. Lyft est une société qui fournit un service similaire à celui d'Uber en rapprochant des personnes qui cherchent à être véhiculées et des conducteurs. Rejetant les demandes, le défendeur fit valoir que les conducteurs pouvaient accéder à la plateforme quand ils le souhaitaient et déterminer librement leur zone géographique d'intervention. Ils utilisaient également leur propre équipement et étaient payés après chaque course effectuée, et non à la semaine ou au mois.

De leur côté, les plaignants faisaient valoir que Lyft contrôlait l'exécution de leur prestation et pouvait mettre fin à la relation à tout moment. En effet, cette société n'hésitait pas à rompre ses engagements contractuels avec des chauffeurs jugés récalcitrants aux directives de Lyft. En effet, le défendeur avait fourni aux chauffeurs des documents expliquant comment effectuer le travail, y compris un guide du conducteur et un rappel des règles du code de la route.

Initialement, la décision devait être rendue par un magistrat professionnel mais le droit américain le permettant, celui-ci refusa de trancher et décida de renvoyer l'affaire devant un jury populaire. Le juge agit de la sorte car selon lui il existait des arguments valables dans les deux camps. D’une part, Lyft exerçait effectivement un pouvoir de contrôle et de sanction sur les travailleurs mais aussi, d'autre part, ces derniers disposaient d'une grande indépendance dans la mesure où ils pouvaient librement déterminer leur temps de travail.

Etait-ce la crainte d'affronter un jury populaire qui amena Lyft à transiger ? Quoi qu'il en soit, le défendeur fit une première offre transactionnelle pour éteindre l'action : le versement de 12.25 millions de dollars. Cette somme fut refusée par le juge car estimée insuffisante. La décision s'explique par le fait que l'action entreprise était une action collective et que le nombre de personnes potentiellement incluses dans le groupe était de 100 000. Rapportée au nombre de bénéficiaires, l'indemnisation offerte fut donc jugée

3 Cotter v. Lyft Inc., $\mathrm{n}^{\circ}$ 13-cv-04065-VC (N.D. Cal.). 
« grossièrement insuffisante ». Le défendeur proposa alors 27 millions et cela fut accepté par le tribunal en juillet 2016, à la condition supplémentaire que Lyft modifie trois choses dans sa gestion des travailleurs:

- Il ne pourrait plus désactiver le compte des chauffeurs de manière arbitraire et ne pourrait le faire que pour certaines raisons communiquées à l'avance ;

- Il devrait mettre en place dans l'entreprise un système de résolution des conflits permettant aux travailleurs de faire valoir leurs réclamations sans devoir recourir à des tribunaux;

- Les chauffeurs pourront bénéficier d'informations supplémentaires sur les passagers avant de prendre la décision d'accepter ou non leur demande de trajet.

Si cette décision semble financièrement satisfaisante pour les requérants, elle ne fut pas de nature à aider les demandeurs subséquents car l'une des contreparties obtenues par Lyft, outre l'abandon de l'action en justice, fut que les chauffeurs n'obtiennent pas la qualité de salariés.

En mars 20154, des travailleurs sous contrat avec Postmates saisirent à leur tour le juge d'une action de groupe sollicitant la requalification de leur relation en salariat et réclamant corrélativement le bénéfice des dispositions légales sur le salaire. Postmates, service de livraison de repas, avait comme Deliveroo recours à des chauffeurs non professionnels qui avaient accepté leur statut initial d'indépendants avant de se raviser devant les difficultés matérielles engendrées par ce statut. Là encore, les parties transigèrent à hauteur de 8.75 millions de dollars et les requérants obtinrent des avantages supplémentaires tel que le bénéfice d'une assurance en cas d'accident de travail. Toutefois, comme dans le cas précité, les demandeurs renoncèrent à la qualification de salariés.

En novembre 20165, une action de groupe fut intentée contre Uber par certains de ses conducteurs qui réclamaient la qualification de salariés. A ce titre, ils revendiquaient l'application du droit du travail new yorkais sur le salaire, impliquant des dommages et intérêts pour non-respect de la législation sur le salaire minimum, le paiement d'heures supplémentaires, de pourboires et le remboursement de frais professionnels. Des raisons procédurales entraînèrent l'échec d'une partie des demandes, de sorte qu'Uber n'eut qu'à indemniser trois chauffeurs à hauteur de 40000 dollars.

\section{II - UNE JURISPRUDENCE PARTAGÉE}

Mais le juge américain ne pouvait être indéfiniment tenu à l'écart de la problématique posée par le statut des travailleurs au moyen de transactions judiciaires. Les premières décisions sur la qualification juridique de ces engagements contractuels ne furent pas favorables aux requérants. Toutefois, la Cour Suprême californienne vient plus récemment de donner espoir aux travailleurs de l'économie collaborative.

4 Singer v. Postmates Inc., $\mathrm{n}^{\circ} 4:$ 15-cv-01284-JSW (N.D. Cal.).

5 Burgos v. Uber Technologies Inc., $\mathrm{n}^{\circ}$ 1: 16-cv-08512 (S.D.N.Y.). 
Au titre des décisions qui rejettent la qualité de salarié, peut être citée celle du juge Michael Baylson du tribunal d'un district de Pennsylvanie, rendue en avril 2018 ${ }^{6}$. Selon cet arrêt les chauffeurs de limousine Uber doivent être considérés comme des travailleurs indépendants. Les requérants, qui avaient groupé leurs demandes au nom de tous les conducteurs de limousine d'Uber, UberBLACK, travaillaient à Philadelphie. S'appuyant sur le droit fédéral (la Loi Fair Labor Standards Act), ils réclamaient le paiement d'heures supplémentaires et l'application de la législation sur le salaire minimum. Ils furent déboutés au motif qu'Uber n'exerçait pas un contrôle suffisant sur ses chauffeurs. Les conducteurs disposaient de l'indépendance nécessaire pour travailler quand ils le souhaitaient, où ils le souhaitaient, et étaient libres de vaquer à leurs occupations personnelles entre les trajets.

On note toutefois une évolution de la position des juges et à cet égard, l'exemple de la jurisprudence californienne est particulièrement pertinent.

La distinction entre un salarié et un travailleur indépendant a été au cœur de nombreux arrêts avant que la solution ne soit tranchée, en Californie, par la Cour Suprême de cet État dans l'arrêt Borello de 1989 relatif à un cultivateur de concombres ${ }^{7}$. Dans cet arrêt, la Cour Suprême californienne a énuméré les facteurs à prendre en considération afin de faire la distinction entre un travailleur indépendant et un salarié. Ce faisceau d'indices se présente sous forme de questions:

- L'employeur fournit-il des outils et un lieu de travail?

- Le travailleur doit-il investir dans l'équipement, le matériel ou les aides?

- Le service exige-t-il une compétence spéciale?

- Le travailleur participe-t-il aux bénéfices et aux pertes de l'entreprise?

- L'activité du travailleur est-elle similaire à celle exercée dans l'entreprise?

- Le travail est-il effectué sous la direction de l'employeur?

De l'ensemble de ces critères se dégagent deux conditions essentielles à la reconnaissance de la qualité de salarié :

- Un travail personnellement exécuté par le salarié en échange d'une rémunération ;

- Un pouvoir de contrôle de l'employeur.

C'est sur le fondement de cette jurisprudence traditionnelle que les premiers juges californiens ont statué lorsqu'ils furent saisis de demandes de requalification en contrat de travail.

Ainsi le 8 février 2018, une Cour de district californienne a débouté un contractant de GrubHub qui revendiquait la qualité de salarié. GrubHub est une entreprise spécialisée dans la livraison de repas qui met en contact des restaurateurs et des clients souhaitant acheter des aliments. La livraison est effectuée par des coursiers, travailleurs indépendants, qui possèdent leur propre véhicule ou vélo. L'un des conducteurs, M. Lawson, intenta des poursuites pour violation de la législation sur le salaire minimum et non-paiement des heures supplémentaires, et sollicita également le remboursement de frais de transport. Ces demandes indemnitaires ne pouvaient aboutir que si le juge accordait la qualité de salarié au requérant. La Cour adopta la solution inverse en raison du faible contrôle exercé par GrubHub sur le travailleur. En effet, la plateforme n'effectuait aucune espèce de contrôle

6 Razak v. Uber Technologies Inc., U.S. District Court for the Eastern District of Pennsylvania, Case $\mathrm{n}^{\circ} 2: 16-\mathrm{cv}-00573$.

7 S. G. Borello \& Sons, Inc. v. Department of Industrial Relations (1989) - 256 Cal. Rptr. 543, 48 Cal. 3d 341,769 P.2d 399. 
sur le travail du demandeur. En outre, aucune directive ne lui était donnée concernant le délai de livraison ou l'itinéraire à emprunter. Enfin, le chauffeur pouvait librement décider de son emploi du temps en désactivant temporairement son compte et en le réactivant lorsqu'il le souhaitait.

Par une décision rendue à l'unanimité le 30 avril 2018', la Cour suprême de cet Etat, créant la surprise, mit un terme à la jurisprudence Borello désormais désuète en adoptant une nouvelle définition du salariat, suffisamment large pour potentiellement englober les travailleurs des plateformes d'économie collaborative. Cette décision, qualifiée de " tremblement de terre ${ }^{9}$ remet potentiellement en cause les modèles d'emploi utilisés par Uber, Lyft et des dizaines d'autres sociétés utilisant des travailleurs indépendants.

Dans son arrêt de 85 pages, la plus haute juridiction de cet État a rejeté le test classique qui permet de distinguer les salariés des travailleurs indépendants au profit d'une définition plus simple, appelée "ABC», déjà utilisée dans le New Jersey et le Massachusetts. En vertu du nouveau test, un travailleur ne peut être considéré comme un entrepreneur indépendant que si trois critères sont remplis:

- l'entreprise est en mesure d'établir que le requérant exerce une maîtrise totale sur la prestation de travail ;

- son travail englobe des tâches qui n'entrent pas dans le champ de compétences habituel de l'entreprise ;

- le travailleur exerce habituellement son activité en toute indépendance dans une entreprise dont l'activité concurrence celle de l'employeur qui l'a embauché.

Le second critère est particulièrement problématique pour les plateformes employeurs car il leur est impossible de prouver que l'activité des livreurs ou des conducteurs est étrangère à l'activité principale de l'entreprise. Ainsi, la livraison est l'activité principale de Deliveroo, de même que la conduite de particuliers est l'activité principale d'Uber. Ce faisant, le juge rend difficile une classification des travailleurs de ces plateformes dans la catégorie des indépendants et ne leur permet plus de s'exonérer de leurs obligations légales d'employeurs.

De l'avis de certains experts ${ }^{10}$, cette décision sera riche de suites judicaires car elle devrait déclencher une avalanche de poursuites en justice contre diverses entreprises qui utilisent des travailleurs faussement qualifiés d'indépendants. Les sociétés acteurs de l'économie participative sont principalement visées, mais elles ne sont pas les seules. A côté d'Uber, des employeurs comme McDonald's et Fedex pourraient être appelés à comparaître devant le juge en raison de ce revirement prétorien.

8 Dynamex Operations West, Inc. v. Superior Court of Los Angeles County.

9 L. Sumagaysay et E. Baron, " Gig economy 'earthquake': California Supreme Court rules on worker classification The Mercury News ", $1^{\text {er }}$ mai 2018, https://www.mercurynews.com/2018/05/01/gigeconomy-earthquake-california-supreme-court-rules-on-worker-classification/

$10 \mathrm{ld}$. 


\section{III - DE POSSIBLES ÉVOLUTIONS}

Cette jurisprudence divergente a amené la doctrine et le législateur à réagir. Tout d'abord, certains auteurs, venant au secours des juges, ont proposé une nouvelle définition du statut de salarié qui correspondrait davantage aux formes modernes d'emploi. Ainsi le professeur Grant Brown ${ }^{11}$ propose un nouveau test qui comporte deux volets :

- Le travailleur peut-il, au moyen de ses compétences, améliorer sa situation économique dans l'entreprise?

- L'activité du salarié recoupe-t-elle celle de l'entreprise?

La première étape de ce test est spécialement adaptée aux travailleurs faiblement qualifiés qui ne disposent d'aucun moyen de progresser hiérarchiquement. Les conducteurs Uber n'ont d'autre horizon professionnel que conduire. En outre, cette absence de compétences spécifiques rend impossible une amélioration de leurs conditions de travail car ils ne sont pas en mesure de négocier les tarifs.

La seconde étape est déjà utilisée par le juge. Elle consiste à vérifier si l'activité du travailleur correspond à celle de l'entreprise.

Une application du nouveau test aux travailleurs des plateformes participatives conduira très certainement à la requalification de leur relation en contrat de travail.

Une autre partie de la doctrine américaine plaide pour la création d'un statut hybride qui emprunterait à la fois à la catégorie du travailleur indépendant et à celle du salarié. En quelque sorte, ces auteurs demandent la création d'une troisième voie. Mme Megan Carboni ${ }^{12}$ utilise la terminologie de "contactant dépendant", tandis que les professeurs Seth Harris et Alan Krueger ${ }^{13}$ préfèreraient l'appellation de «salariés indépendants ». Ces travailleurs atypiques, sans pour autant pouvoir revendiquer tous les droits des salariés, devraient se voir reconnaître certaines de leurs prérogatives, notamment le droit de se syndiquer et celui de la négociation collective.

Cette proposition ne rencontre, pour l'instant, aucun succès. II n'appartient pas au juge d'effectuer un changement aussi conséquent et le législateur, plutôt que cette troisième voie, est intervenu en classant les travailleurs des plateformes collaboratives dans la catégorie de travailleurs indépendants. La Caroline du Nord, I'Arkansas, et I'Indiana sont intervenus dans ce sens ${ }^{14}$.

D'autres autorités publiques, sans se prononcer sur la qualification de ces travailleurs, leur ont accordé des droits supplémentaires immédiatement contestés par les plateformes collaboratives. Ainsi la ville de Seattle, en décembre 2015, a adopté une ordonnance

11 G. E. Brown, "An Uberdilemma: Employees And Independent Contractors In The Sharing Economy ", Maryland Law Review, 2006, vol. 75, p. 15.

12 M. Carboni, "A New Class of Worker for the Sharing Economy ", Richmond Journal of Law \& Technology, 2016, vol. 22, n² 4, p. 1.

13 S. D. Harris, A. B. Krueger, « A Proposal for Modernizing Labor Laws for Twenty-First-Century Work: The 'Independent Worker'», The Hamilton Project, Discussion Paper 2015-10, 2015, http://www.hamiltonproject.org/assets/files/modernizing labor laws for twenty first century work krueger harris.pdf

14 T. Devlin, S. Chiu, «Is Your Uber Driver or Lyfter an Employee or Independent Contractor and Why Does It Matter? ", Westlaw's Secondary Source Analytical Content, Emerging Areas of Practice Series, 2018. 
autorisant les conducteurs d'Uber à se syndiquer. L'idée justifiant cette mesure est de mettre ces travailleurs atypiques en position de négocier collectivement une amélioration de leur rémunération. Cette ordonnance fit l'objet d'un recours à l'initiative de la Chambre de commerce américaine qui compte Uber et Lyft parmi ses adhérents. Elle fit valoir qu'en permettant aux conducteurs de négocier leur salaire, qui est basé sur les tarifs imposés aux passagers, la ville de Seattle leur permettrait de fixer les prix en violation de la législation fédérale antitrust. Le premier juge la débouta mais en appel, la requérante obtint gain de cause. Le 11 mai 2018, une Cour d'appel fédérale basée à San Francisco infirma le jugement en considérant que la ville de Seattle avait enfreint le droit fédéral ${ }^{15}$.

Pour conclure, même si certains auteurs américains déplorent les effets " néfastes » du droit du travail sur l'économie collaborative ${ }^{16}$, d'autres soulignent ironiquement que cette économie parfois décrite de manière flatteuse comme une économie de partage l'est assez peu par nature. Cette expression d'«économie de partage» est peu appropriée pour un secteur qui est fondamentalement commercial et pas centré sur la générosité ou l'altruisme ${ }^{17}$. De même, l'apparente liberté laissée aux travailleurs se transforme vite en dépendance économique envers une plateforme puissante contre laquelle seul le droit des contrats est en mesure de fournir une aide aux cocontractants les plus vulnérables.

Le droit du travail est sans influence dès lors que ces travailleurs ne sont pas en mesure de convaincre un juge qu'ils ont la qualité de salariés.

La création d'un statut spécifique aux travailleurs des plateformes collaboratives est une option mais elle rencontre deux difficultés : d'une part, il faudrait déterminer un socle minimal de protection dont bénéficieraient à la fois les salariés et ces travailleurs atypiques. D'autre part, cette solution ne rencontre qu'un enthousiasme très modéré si on excepte une partie de la doctrine. Ainsi, lors du Sommet de 2015 de la Maison-Blanche sur la voix des travailleurs, le président Obama a mis en garde contre la création d'une troisième catégorie de travailleurs qui, selon lui, ne recevraient qu'une «version allégée des protections et des droits fournis par un syndicat ${ }^{18}$.

Dans l'attente d'une intervention législative qui résoudrait ce problème, la jurisprudence américaine en est réduite à utiliser des définitions, parfois anciennes, de la relation de travail afin de se prononcer sur le statut de ces travailleurs particuliers. Toutefois, même si elle n'est pas unanime, la jurisprudence fait parfois preuve d'audace, à l'image de la Cour Suprême de Californie qui a renversé un courant prétorien bien établi pour faire entrer les collaborateurs de ces plateformes dans la catégorie des salariés.

15 U.S. Chamber of Commerce v. City of Seattle, 9th U.S. Circuit Court of Appeals, n¹7-35640.

16 E. Atmore, "Killing the Goose That Laid the Golden Egg: Outdated Employment Laws Are Destroying the Gig Economy », Minnesota Law Review, 2017, vol. 102, n², p. 887.

17 S.-Y. Oei, «The Trouble with Gig Talk: Choice of Narrative and the Worker Classification Fights », Law and Contemporary Problems, 2018, vol. 81, n 3, p. 107.

18 T. Devlin, S. Chiu, «Is Your Uber Driver or Lyfter an Employee or Independent Contractor and Why Does It Matter?», op. cit. 